\title{
Design expert as a statistical tool for optimization of 5-ASA-loaded biopolymer- based nanoparticles using Box Behnken factorial design
}

\author{
Wasim Akram ${ }^{*}$ and Navneet Garud
}

\begin{abstract}
Background: The overall objective was to prepare a highly accurate nanocarrier system of mesalamine for the treatment of ulcerative colitis with increased therapeutic efficacy and targeting. In the formulation of nanocarrier systems, optimization is a critical process for understanding nanoformulation variables and quality aspects. The goal of the present work was to determine the effect of independent variables, i.e., the concentrations of chitosan, carboxymethyl inulin (CMI), and the drug on the response variables, i.e., particle size and percent entrapment efficiency of the mesalamine-loaded nanoparticle using the Box Behnken design (BBD). The correlation between the independent and dependent variables was investigated using the Design Expert generated mathematical equations, contour, and response surface designs.

Result: An optimized batch was developed using the ionotropic gel method with selected independent variables (A: + 1 level, B: 0 level, C: -1 level) and the developed nanoparticles had a particle size of $184.18 \mathrm{~nm}$, zeta potential $26.54 \mathrm{mV}$, and entrapment efficiency $88.58 \%$. The observed responses were remarkably similar to the predicted values. The morphological studies revealed that the formulated nanoparticles were spherical, and the results of the FTIR and DSC studies indicated the drug-polymer compatibility. The nanoparticle showed less than $5 \%$ release in the $\mathrm{pH}$ 1.2. In the colonic region ( $\mathrm{pH}$ 7.4), more than $80 \%$ of the medication was released after $24 \mathrm{~h}$. The kinetics study showed that the Higuchi and Korsemeyer-Peppas models had $R^{2}$ values of 0.9426 and 0.9784 respectively, for the developed formulation indicating linearity, as revealed by the plots. This result justified the sustained release behavior of the formulation.

Conclusion: The mesalamine-loaded chitosan-CMI nanoparticle has been successfully developed using the ionotropic gelation method. The nanoparticles developed in this study were proposed to deliver the drug to its desired site. The developed nanoparticles were likely to have a small particle size with positive zeta potential and high percent drug entrapment. It could be stated from the results that BBD can be an active way for optimizing the formulation and that nanoparticles can be a potential carrier for delivering therapeutics to the colon.
\end{abstract}

Keywords: Biopolymers, Colon targeting, lonotropic gelation, Nanoparticles

* Correspondence: wasimjiwaji@gmail.com

School of Studies in Pharmaceutical Sciences, Jiwaji University, Gwalior, M.P,

India 


\section{Background}

Ulcerative colitis (UC) and Crohn's disease (CD) are two major inflammatory bowel disorders (IBDs), which are a group of chronic gastrointestinal (GI) conditions that can affect intestines (both small and large) and are most frequent in adolescents and young adults between the ages of 15 and 30 [1]. Diarrhea, stomach pain, weight loss, rectal bleeding, fatigue, and fever are clinical signs. Since there are no effective treatments, the most successful protocols depend on long-term remission to prevent relapse [2].

5-ASA (5-Aminosalicylic acid) is a commonly used drug for an active CD or UC and for maintaining symptom remission in UC. Although the mechanism of action is unknown, some research findings specify that 5-ASA shows its action locally on the intestinal mucosa. On the other hand, 5-ASA is rapidly and extensively absorbed in the upper GI tract when taken orally, resulting in low bioavailability. This necessitates a high daily dose of 5ASA, which can cause systemic side effects, including interstitial nephritis, blood dyscrasia, pancreatitis, and pleuropericarditis [3]. In the current scenario, designing and delivering 5-ASA with increased bioavailability, effectiveness, and reduced side effects is a top priority.

Nanotechnology has been investigated as a potential method for improving the therapeutic efficacy and bioavailability of 5-ASA by using it to design and deliver anti-inflammatory drugs. Nanoparticles can be used to deliver therapeutics directly to the site of inflammation, avoiding drug-related systemic side effects. Nanoparticles gained much popularity for playing a significant role in designing oral sustained or controlled release solid dosage form because of their potential benefits viz., reduced risk of systemic toxicity, reduced risk of local irritation, predictable gastric emptying, increased bioavailability, improved reproducible pharmacokinetic profile, improved stability and patient compliances too $[4,5]$. Furthermore, regulated and site-specific drugcarrier systems have been prepared to improve the therapeutic bioavailability at the desired site. Numerous methods have been used to convey therapeutics directly to the desired site, one of which is the 5-ASA entrapment of the charged carrier [6], which has the added benefit of favoring mucoadhesion and possibly cell absorption of positively charged carrier system through their interaction with negative cell membranes. Bioadhesion to cell membrane can be advantageous for sitespecific delivery because it allows for stronger mucosal surface contact for drug absorption and release. Increased intestinal motility, which is common in IBD, can decrease nanocarrier clearance. Mucoadhesiveness may prevent 5-ASA from being absorbed in the upper GI tract, lowering the dose required to achieve a therapeutic effect. Consequently, there is a decrease in side effects, implying an improvement in patient adherence [7]. Drug delivery carriers with nanometer-sized dimensions presented increased transit time in inflamed colonic regions, and offered extra assistance for IBD care. By using the augmented epithelial permeability and retention (eEPR) effect, it is possible to increase and specifically deliver drug molecules to the colitis tissue, and [8] allows the preferential absorption of nanoparticles by immune cells, which are increased significantly in the inflamed areas [9]. By decreasing the particle size, it may be feasible to evade quick carrier removal through diarrhea, a frequent sign of IBD. Nanosystems are easily absorbed into inflamed tissues and cells, avoiding carrier removal [10].

From the previous researcher findings, chitosan was considered as a vital molecule to examine these hypotheses, a biopolymer with favorable biocompatible and biodegradable properties, as well as to facilitate membrane permeability and quickly deteriorates in serum by lysozyme, Chitosan is a polysaccharide that is widely used in the delivery of drugs to its desired site, and it is used in nano- and micron-sized particulate matter. Its notable characteristics comprise bioadhesion and potential for drug delivery [11], as well as negatively charged polysaccharides, which help to shape more complex nano vehicles. Another polymer, the carboxymethyl inulin (CMI), a derivative of inulin, was employed to develop chitosanCMI composite nanoparticles. Inulin is a polysaccharide made up of repeated fructose units used in the pharmaceutical industry because it is biocompatible and chemically simple to modify, despite being exogenous to the human body. It also has poor absorption in most of the tissues and can quickly filter through the kidneys. It also has characteristics in common with CMI, such as being readily available and inexpensive and having non-toxic metabolites [12].

A very little research has been done on the delivery of 5-ASA to a specific site using a biopolymer-decorated nanoparticulate system. In our research, we used response surface methodology (RSM) and a BBD to optimize the entrapment efficiency (EE) and particle size of 5-ASA-loaded nanosystem composed of CMI and chitosan, the two features that have a major impact on the success of the dosage type, bioavailability at the desired site, and drug efficacy $[13,14]$.

$\mathrm{BBD}$ is a design of surface response with many advantages over other experimental designs. In this, only three levels of independent variables must be run, and the variation of the expected result at any point is determined solely by its distance from the design center point [15].

Since our goal was to optimize the size of the particle and percent entrapment efficiency of biopolymer-based 5-ASA nanoparticles simultaneously, we combined the 
two separate responses into a single composite system using multi-criteria decision analysis (MCDA). MCDA is a formal technique to decision-making that allows for selecting of alternatives based on various parameters while avoiding the drawbacks of unstructured decisionmaking.

There have been no studies on the fabrication or optimization of 5-ASA nanoparticles using chitosan and CMI to our knowledge. Even though there were several kinds of literature on the optimization of nanoparticles containing various agents, none of them utilized the RSM technique to optimize chitosan-CMI nanoparticles.

\section{Methods}

\section{Material}

Mesalamine (5-ASA) was obtained from Zydus Healthcare Pvt. Ltd. (Ahmedabad, India). Chitosan (Purified viscosity grade, $50 \mathrm{cps}$, MW $150 \mathrm{KDa}$ ) was purchased from CDH Laboratory Pvt. Ltd. (Mumbai, India). Inulin was procured from Amruta Herbals Pvt. Ltd. All the other chemicals and reagents used in this study were of commercial grade and were used precisely as received, with no further purification.

\section{Experimental design}

Employing Stat-Ease Design-Expert Software, a 15-set BBD with 3-independent factors with 3-level was preferred to optimize the 5-ASA decorated chitosan-CMI nanoparticulate system (Version 11.1.0.1 Stat-Ease, Inc., Minneapolis, MN 55413). The 3 independent factors include the concentration of the drug, $A$; chitosan, $B$; and of CMI, $C$, which are thought to be important factors in the production of nanoparticles. As shown in Table 1, these factors are analyzed at the levels of low $(-1)$, medium (0), and strong $(+1)$. Entrapment performance and particle size are two dependent variables. The nonlinear quadratic model equation shown below can be used to calculate the expected $Y$ response:

$$
\begin{aligned}
Y= & \alpha_{0}+\alpha_{1} A+\alpha_{2} B+\alpha_{3} C+\alpha_{1} \alpha_{1} A^{2}+\alpha_{2} \alpha_{2} B^{2} \\
& +\alpha_{3} \alpha_{3} C^{2}+\alpha_{1} \alpha_{2} A B+\alpha_{1} \alpha_{3} A C+\alpha_{2} \alpha_{3} B C
\end{aligned}
$$

where $Y$ is the calculated response; $\alpha_{0}$ is the intercepts; $\alpha_{1}, \alpha_{2}, \alpha_{3}$ are the coefficients in linear form; the interception is $\alpha_{1} \alpha_{1}, \alpha_{2} \alpha_{2}, \alpha_{3} \alpha_{3}$; and the quadratic coefficients are $\alpha_{1} \alpha_{2}, \alpha_{1} \alpha_{3}$, and $\alpha_{2} \alpha_{3}$.

\section{Formulation of chitosan-CMI nanoparticles}

Chitosan-CMI nanoparticles were formed by ionotropic gelation of the positively charged amino group of chitosan with the negatively charged carboxyl group of CMI, as shown in Fig. 1. Chitosan was dissolved in $1.0 \%(\mathrm{v} / \mathrm{v})$ lactic acid solution at a range of $2.0 \mathrm{mg} / \mathrm{mL}$, and $\mathrm{pH}$ was changed to 4.0 by slowly adding $1.0 \mathrm{M} \mathrm{NaOH}$ until full dissolution. PBS ( $\mathrm{pH}$ 7.4) was used to dissolve CMI at a range of $0.5 \mathrm{mg} / \mathrm{mL}$. The correct amount of 5-ASA was applied to the CMI solution and fully dissolved. Chitosan-CMI nanoparticles were formed spontaneously by adding $100 \mathrm{~mL}$ CMI of dissolved drug solution to $100 \mathrm{~mL}$ chitosan solution $(10 \mathrm{~mL} / \mathrm{min})$ at room temperature while stirring at $2000 \mathrm{rpm}$ (RQT-124A/D stirrer, Remi motors, India). After 5 min of stirring, the solution had become slightly cloudy, and the stirring was continued for another $20 \mathrm{~min}$ to stabilize the chitosanCMI nanoparticle framework. The solution was centrifuged for $20 \mathrm{~min}$ at $6000 \mathrm{rpm}$ (R-8C / RM-12C Centrifuge machine, Remi motors, India). The precipitate was discarded, and the supernatant was filtered through a 1.0- $\mu \mathrm{m}$ membrane filter (Millipore, Omnipore Membrane) to obtain chitosan-CMI nanoparticle suspension. They were lyophilized for $48 \mathrm{~h}$ to get nanoparticles in powder form.

\section{Optimization employing Box Behnken design}

Box Behnken statistical design is an efficient method for optimizing a system in a short amount of time. BoxBehnken design (BBD) was used to optimize mesalamine decorated chitosan-CMI nanoparticles employing Design Expert Software Version 11.1.0.1 (Stat-Ease Inc., USA). The concentrations of chitosan (factor $A$ ), CMI (factor $B$ ), and drug (factor $C$ ) were chosen as independent variables at two low and high levels respectively, shown in Table 1. Particle size and percent drug entrapment were chosen as response factors. Table 2 depicts the pattern of the design. Various statistical parameters such as the probability value ( $p$-value), the regression coefficient $\left(R^{2}\right.$ value), the Fisher model value ( $F$ value), and the lack of fit $F$ value were used to adapt the responses to the appropriate mathematical model developed by design. In addition to the best fit model, quadratic polynomial response equations with key factors and interaction factors have been developed. The presented model's suitability and validity were assessed using ANOVA. The optimal formulation was chosen based on response variables'

Table 1 Levels and the experimental condition for Box Behnken design

\begin{tabular}{lllll}
\hline Factor & Designation & Low level $(-)$ & Mid-level (0) & High level (+) \\
\hline A & Concentration of drug $(\% \mathrm{w} / \mathrm{v})$ & 0.1 & 0.3 & 0.5 \\
B & Concentration of chitosan $(\% \mathrm{w} / \mathrm{v})$ & 0.1 & 0.15 & 0.2 \\
C & Concentration of carboxymethyl inulin (CMI) $(\% \mathrm{w} / \mathrm{v})$ & 0.06 & 0.08 & 0.1 \\
\hline
\end{tabular}




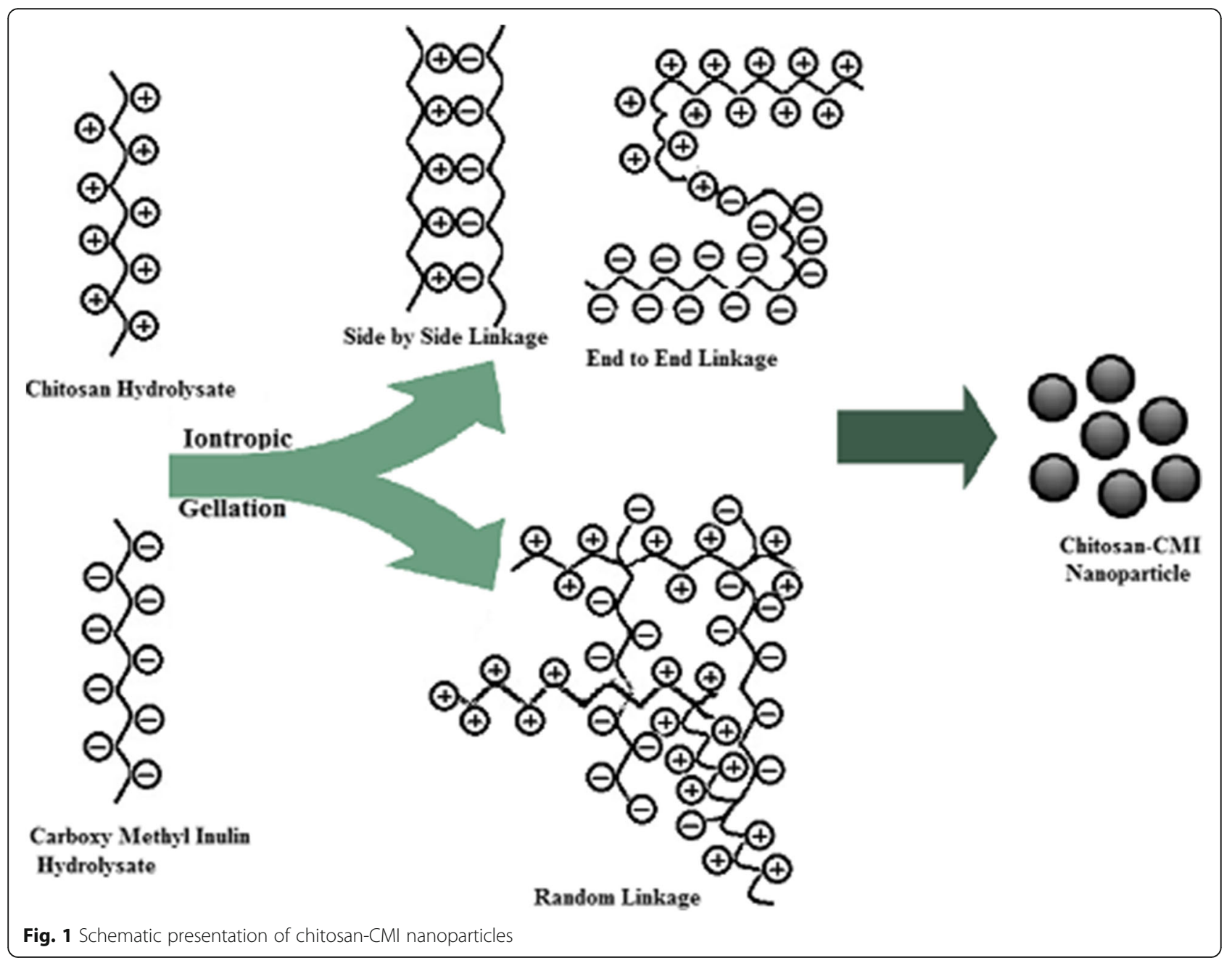

Table 2 Experimental runs for Box-Behnken design

\begin{tabular}{llllll}
\hline Formulation & $\begin{array}{l}\text { Factor } \mathbf{A} \\
\text { Concentration of chitosan } \\
\% \text { w/v }\end{array}$ & $\begin{array}{l}\text { Factor B } \\
\text { Concentration of CMI } \\
\% \text { w/v }\end{array}$ & $\begin{array}{l}\text { Factor C } \\
\text { Concentration of drug } \\
\% \text { w/v }\end{array}$ & $\begin{array}{l}\text { Response } \mathbf{1} \\
\text { Particle size } \\
\text { nm } \pm \text { SD }\end{array}$ & $\begin{array}{l}\text { Response } 2 \\
\text { Entrapment efficiency } \% \\
\text { nm } \pm \text { SD }\end{array}$ \\
\hline 1 & $0.2(+1)$ & $0.08(0)$ & $0.5(+1)$ & $199.25 \pm 1.2$ & $79.20 \pm 2.2$ \\
2 & $0.15(0)$ & $0.08(0)$ & $0.3(0)$ & $179.41 \pm 2.2$ & $72.32 \pm 3.3$ \\
3 & $0.08(0)$ & $0.5(+1)$ & $160.36 \pm 3.0$ & $59.36 \pm 4.5$ \\
4 & $0.1(-1)$ & $0.06(-1)$ & $0.3(0)$ & $15,535 \pm 3.6$ & $66.14 \pm 5.2$ \\
5 & $0.1(-1)$ & $0.1(+1)$ & $0.5(+1)$ & $188.85 \pm 4.5$ & $64.25 \pm 5.0$ \\
6 & $0.15(0)$ & $0.08(0)$ & $0.1(-1)$ & $151.62 \pm 1.5$ & $66.22 \pm 3.6$ \\
7 & $0.1(-1)$ & $0.06(-1)$ & $0.3(0)$ & $208.55 \pm 1.8$ & $86.50 \pm 1.4$ \\
8 & $0.2(+1)$ & $0.1(+1)$ & $0.3(0)$ & $192.55 \pm 1.5$ & $77.45 \pm 1.5$ \\
9 & $0.2(+1)$ & $0.1(+1)$ & $0.1(-1)$ & $191.27 \pm 2.0$ & $71.48 \pm 2.0$ \\
10 & $0.15(0)$ & $0.1(+1)$ & $0.3(0)$ & $174.22 \pm 2.8$ & $55.34 \pm 3.5$ \\
11 & $0.1(-1)$ & $0.06(-1)$ & $193.32 \pm 4.2$ & $73.38 \pm 1.8$ \\
12 & $0.15(0)$ & $0.08(0)$ & $0.5(+1)$ & $180.20 \pm 5.2$ & $73.49 \pm 1.6$ \\
13 & $0.15(0)$ & $0.08(0)$ & $0.1(-1)$ & $184.18 \pm 2.5$ & $88.58 \pm 1.0$ \\
14 & $0.2(+1)$ & $0.08(0)$ & $0.3(0)$ & $178.42 \pm 1.8$ & $72.25 \pm 2.2$ \\
15 & $0.15(0)$ & $0.06(-1)$ & $0.1(-1)$ & $179.34 \pm 3.6$ & $82.54 \pm 2.6$ \\
\hline
\end{tabular}


desirability, and it was tested physicochemically, in vitro, and ex vivo.

\section{Particle size and size distribution}

With the help of dynamic laser scattering, the size distribution and average particle size of 5-ASA loaded nanoparticles were calculated using a Zetasizer (Nano ZS, Malvern Instruments Ltd., UK). Nanosuspensions were properly diluted with distilled water and homogenized for $2 \mathrm{~min}$ to form a homogeneous dispersion before being placed in a quartz cell. With He-Ne laser, the hydrodynamic diameter of the particles was estimated at a scatter angle of 900 at $25{ }^{\circ} \mathrm{C}$. All the samples were measured in triplicate, and the outcomes revealed as the average particle size \pm SD.

\section{Zeta potential}

The electrophoretic light scattering approach was used to determine the zeta potential of the prepared formulation (Nano-ZS ZEN 3600, Malvern Instruments Ltd., UK). The nanoparticles in water were diluted and stabilized at $25{ }^{\circ} \mathrm{C}$ before being put in transparent one-use zeta cubicles. Cataphoretic kinesis among the electrodes was used to achieve zeta potential. The tests were carried out three times, and the findings were measured in millivolts $(\mathrm{mV})$.

\section{Surface morphology}

The microstructure of the prepared formulation loaded with 5-ASA was studied using a transmission electron microscope (TEM). The nanoparticles were diluted with sterile deionized water sonicated for $2 \mathrm{~min}$ in a sonicator, and a drop placed on a carbon-coated copper mesh and viewed using TEM (JOEL H-7500, Hitachi, Japan).

\section{Drug content}

A $100.0 \mathrm{mg}$ quantity of each batch of the chitosan-CMI nanoparticles powder was digested in $250 \mathrm{~mL}$ of buffer $(\mathrm{pH} 7.4)$ in a 250-mL graduated cylinder with intermittent shaking for $24 \mathrm{~h}$ at $37 \pm 0.5{ }^{\circ} \mathrm{C}$ to fully dissolve the encapsulated drug. A high-speed mechanical magnetic stirrer was used to stir the solution for $25 \mathrm{~min}$ at 2500 rpm (RQT-124A/D stirrer, Remi Motors, India). The solution was then filtered to remove dissolved polymers and debris using Whatman ${ }^{\odot}$ filter paper (\#41). Aliquots of the clear supernatant fluid were used to test the actual drug content using a UV-visible spectrophotometer (Shimadzu 1800, Japan) against the buffer as blank. Each analysis was conducted thrice, and the results expressed as means \pm standard deviation. The following formula was used to calculate the drug entrapment efficiency (DEE):

$$
\operatorname{DEE}(\%)=\frac{\text { Percent drug loading }}{\text { Theoretical drug loading }} \times 100
$$

\section{Fourier-transform infrared (FTIR) spectroscopy}

The $\mathrm{KBr}$ disc method was used to record spectra of pure drug, CMI, chitosan, and drug-loaded CMIchitosan nanoformulation to determine potential chemical interactions between drug and polymer components. The nanoparticles were mixed with FTIR grade anhydrous $\mathrm{KBr}$ (Merck IR Spectroscopy grade) at a ratio of $1: 100$ before being dried at $40 \pm 0.5^{\circ} \mathrm{C}$ to remove all moisture. The mixture was crushed in a mortar with a pestle and sieved, then compressed into a disc, and eventually packed into a sample holder. At ambient temperature, the spectra were scanned using an FTIR spectrometer (1800, Shimadzu, Japan) at a resolution of $4 \mathrm{~cm}^{-1}$ and speed of $1 \mathrm{~cm} / \mathrm{s}$ over a wavelength region of 4000 to $400 \mathrm{~cm}^{-1}$.

\section{Differential scanning calorimeter (DSC)}

A thermal examination of the samples was conducted using a Shimadzu 60 plus-Differential Scanning Calorimeter (DSC) (Shimadzu Technologies, India) to comprehend the potential interactions between the constituents of the formulation. The temperature and energy scales of the calorimeter were calibrated using $\mathrm{Al}_{2} \mathrm{O}_{3}$ as the primary standard. Approximately $8 \mathrm{mg}$ of $\mathrm{CMI}$, chitosan, and CMI-chitosan nanoparticles were heated at a rate of $10{ }^{\circ} \mathrm{C} / \mathrm{min}$ in $40 \mathrm{~mL}$ aluminum pans at a temperature limit of 40 to $400{ }^{\circ} \mathrm{C}$. Thermal examinations were performed in a nitrogen environment at a flow speed of $60 \mathrm{~mL} / \mathrm{min}$. The DSC thermogram of the sample was recorded using Shimadzu software.

\section{In vitro drug release profile of nanoparticles}

Different buffers, like $0.1 \mathrm{~N} \mathrm{HCl}(1.2 \mathrm{pH})$, phosphate buffer (pHs 6.8 and 7.4), were used to perform in vitro drug release studies to mimic the stomach, intestinal, and colon GI conditions. The drug release time profile analysis was conducted on each of the formulations (F1F15). Drug release tests lasted up to $24 \mathrm{~h}$ a day. Samples were removed at different periods. The samples were taken every hour for up to $2 \mathrm{~h}$ in acidic conditions (1.2 $\mathrm{pH})$. After that, at a $\mathrm{pH}$ of 6.8 , sampling was done every 1 to $4 \mathrm{~h}$, and then in colonic condition $\mathrm{pH}, 7.4$ samples were taken for 1 to $10 \mathrm{~h}$; after that, two samples were withdrawn at $12 \mathrm{~h}$ and $24 \mathrm{~h}$. Fresh media was used to replace the samples that had been withdrawn from the system. The drug release from the developed nanoparticle was tested using a dialysis bag (Himedia labs, cutoff weight 12,000-11,000 Da). The dialysis bag was saturated in deionized water overnight before the experiment in this process. A 2-mL nanoparticle dispersion is 
mounted in the $2000 \mathrm{Da}$ dialysis bag, and clamps are used to secure the two ends. The bags were placed in $250 \mathrm{~mL}$ of dissolution media held at $35 \pm 0.5^{\circ} \mathrm{C}$ to determine the drug's release. At predetermined intervals, samples were removed for approximately $24 \mathrm{~h}$. At regular intervals, $1.0 \mathrm{~mL}$ of the sample was withdrawn by adding fresh buffer/fresh dissolution fluid. UV spectrophotometry at $330 \mathrm{~nm}$ was used to examine the samples. All measurements have been done in triplicate. Based on $R^{2}$ and ' $n$ ' values, various kinetic models were used to evaluate the release mechanism of the drug.

\section{Modeling and comparison of in vitro release profiles}

In vitro drug release data was integrated into different kinetic equations to elucidate the mechanisms of drug release from each batch of the formulation [16]. The results were adjusted using zero-order, first-order, HixsonCrowell, Higuchi, and Korsemeyer-Peppas models. The $R^{2}$ determination coefficient must be used to select the "right model" or "goodness-of-fit" criterion for drug dissolution or release phenomenon analysis. An equation to direct this model: $\mathrm{Mt}=M_{0}+K_{0} t$, where Mt represents the quantity of drugs dissolved in time $t, M_{0}$ represents the initial quantity of drugs in the solution, and $K_{0}$ represents the zero-order release rate constant. The rates of drug release from the systems are concentrationdependent, according to first-order. The mathematical equation for this model is $M=M_{0} e k t$, where $M$ represents the quantity of released drug in time $t, M_{0}$ represents the initial quantity of drug in the solution, and $k$ represents the rate constant. Drugs are released from an insoluble matrix as a square root of time, as per the Higuchi model, based on Fickian diffusion. An equation to direct this model: $M=K t$, where $M$ represents the sum of the released drug in particular time $t, K$ represents the respective equation's release rate constant, and $t$ represents the time of release. The Hixson-Crowell model states that drug release is proportional to the variation in particle surface area and diameter, as shown by the equation: $M 1 / 3=K t+M_{0} 1 / 3$, where $M$ represents the quantity of released drug in time $t, M_{0}$ represents the initial quantity of drug in the solution, and $K$ represents the rate constant.

The Korsemeyer-Peppas model is commonly used when the release mechanism is unknown or when more than one type of release phenomenon is considered to study release from the device. $\mathrm{Mt} / \mathrm{M}$-polymer $=K t_{n}$ is the model's equation, where $\mathrm{Mt} / \mathrm{M}$-polymer denotes the percentage of drug released in time $t$ and $K$ denotes the steady integration of the drug/polymer system's structural and geometric characteristics, and $n$ seems to be the release exponent, which denotes mechanism of drug release. Calculation of the $R^{2}$ (correlation coefficient) values compared the accuracy and predictability of these models. The Korsemeyer-Peppas model was employed to differentiate between the competing mechanisms of drug release: Fickian release (distribution facilitated), non-Fickian release (anomaly passage), and case-II transport (release based on relaxation). The Fickian diffusion method of drug release is used when $n$ is less than 0.5 . Non-Fickian release or anomalous transport is suggested by a value of $0.5<n<1.0$. The feature is zero-order release or case-II transport if $n=1$ and super case-II transport if $n>1.0$. The best-suited model was found by evaluating the $R^{2}$ values.

\section{Ex vivo mucoadhesion study}

Prior to the ex vivo analysis, chitosan-CMI nanoparticles $(10 \mathrm{mg})$ were incubated in $1.0 \mathrm{~mL}$ of ethanol containing $1 \% \mathrm{w} / \mathrm{v}$ fluorescein isothiocyanate solution for $24 \mathrm{~h}$. In an ex vivo study, Wistar rats $(220 \mathrm{~g})$ were fasted overnight, provided free access to water, and handled according to CPCSEA guidelines. A warm Tyrode fluid $\left(37^{\circ} \mathrm{C}\right)$ was used to clean the colon to extract lumen material before being sacrificed. Each animal's colon was divided into two equal parts. One part was immersed in a $20 \mathrm{mg}$ fluorescent nanoparticle solution, i.e., OF1 in $10 \mathrm{~mL}$ Simulated colonic fluid (SCF) ( $\mathrm{pH} 7.4$ ), while another part was immersed only in a fluorescent marker. Both parts were subsequently incubated in the gaseous oxygen solution of Tyrode at $37^{\circ} \mathrm{C}$ in the dark. The colonic segments were washed three times in ice-cold SCF to extract any remaining nanoparticles and examined under a fluorescent microscope after $2 \mathrm{~h}$ (Germany's Carl Zeiss) [17]. The study was approved by the Institutional Animal Ethics Committee (IAEC/JU/48).

\section{Results}

\section{Optimization of chitosan-CMI nanoparticles process} variables

The biggest challenge in developing pharmaceutical products and processes is finding the right combination of process variables that will result in high-quality products. With fewer experimental sequences and less time, biostatistical experimental designs may potentially support studying the effects of individual process variables and their interactions [18]. The present study used BBD optimization to accurately measure response variables and create polynomial equations based on experimental results. Appropriate statistical trials were run, the best fit model was chosen, and independent variables that could generate optimal responses were further determined.

Design Expert Version 11.1.0.1 provided 15 test formulations for BBD; the result of particle size and drug entrapment efficiency for all the 15 formulations is summarized in Table 2. Heavy dependence on dependent variables has been reported for the adopted independent 
variables. For all of the response variables, the results were expressed as polynomial equations. By treating the third factor as constant, 3D graphs were used to describe the product parameter interaction.

\section{Effect of formulation variables on particle size}

The $F$-value of the model is high 77.75 , indicating that it is significant. Just $0.01 \%$ of the time does noise causes such a strong $F$-value. The model expressions are important if the Prob $>F$ value below 0.05 [19]. The model expressions $A, C, A B, B C, A^{2}$, and $B^{2}$ are important in this case as shown in Table 3 . When the values are higher than 0.1000 , model expressions are not meaningful. A model contraction is needed to improve the model if it includes a large number of insignificant model expressions. This is not necessary in this case since most of them are less than 0.100 , indicating that factors A and $B$ have the most significant impact on particle size and that factor $C$ has little impact. The $7.75 F$ value of lack of fit indicates that the lack of fit is not substantial with the pure error. In order to get an acceptable model, there must be an insignificant lack of fit. The 0.9801 value of adjusted $R^{2}$ is equal to the predicted $R^{2} 0.8942$. The signal-to-noise ratio is measured by adequate precision. It is preferable to have a ratio of more than four. Our signal-to-noise ratio of 29.907 represents a good sign. The design area can be navigated using this model. For this model, the polynomial equation obtained is as follows:

Particle size $=179.00+17.87 \mathrm{xA}+1.25 \mathrm{xB}+4.38 \mathrm{xC}-8.75 \mathrm{xAB}+$ 1.50xAC-4.25xBC- $5.50 \mathrm{xA}^{2}+8.75 \mathrm{xB}^{2}+0.000 \mathrm{xC}^{2}$

Response predictions for the given levels of each factor can be calculated using the equation in terms of coded factors. The main effects on particle size in the above regression equations are $A, B$, and $C$. The interactive expressions $A B, A C, B C, A^{2}, B^{2}$, and $C^{2}$ represent a nonlinear relationship between responses. As can be seen from the equation and Fig. 3, as the polymer ratio rises from low to high, the particle size increases as well. This could be due to a higher amount of polymer causing increased viscosity which was in acceptance according to the literature $[20,21]$. The formulations have particle sizes ranging from 151.62 to $208.55 \mathrm{~nm}$; the optimized batch average particle size is displayed in Figs. 2 and 3.

\section{Zeta potential}

The zeta potential/surface charge of developed nanoparticles is affected by the polyelectrolyte ratio. The zeta potential is measured to determine the surface charge of the nanoparticles. The zeta potential of nanoparticles strongly depends on the particle's composition and the dispersion medium, and it also indicates the particle's electrical potential. Table 4 shows the individual zeta potential and PDI values for each formulation. Within the ranges of $18.98-36.58$ and $0.232-0.355$ respectively, Fig. 4 shows the zeta potential of the optimized batch. For all the nanoparticles, it was discovered that they have a positive charge value, with the formulation F2 having the highest charge value of 36.58, whereas PDI 0.248 indicates good stability and dispersion homogeneity [22].

\section{Surface morphology}

Advanced techniques such as TEM were used to perform microscopic analysis of the optimized formulation. The particles were found to be spherical with a uniform size range, confirming their size range within the nanolimit in Fig. 5 and revealed that they had a narrow size distribution range.

Table 3 Particle size statistical analysis result

\begin{tabular}{llllll}
\hline Source & Sum of squares & df & Mean square & $\boldsymbol{F}$-value & $\boldsymbol{p}$-value \\
\hline Model & 3533.68 & 9 & 392.63 & 77.75 & $<0.0001$ \\
A-CH & 2556.12 & 1 & 2556.12 & 506.16 & $<0.0001$ \\
B-CMI & 12.50 & 1 & 12.50 & 2.48 & 0.1765 \\
C-5-ASA & 153.13 & 1 & 153.13 & 30.32 & 0.0027 \\
AB & 306.25 & 1 & 306.25 & 60.64 & 0.0006 \\
AC & 9.00 & 1 & 9.00 & 1.78 & 0.2394 \\
BC & 72.25 & 1 & 72.25 & 14.31 & 0.0129 \\
A $^{2}$ & 111.69 & 1 & 111.69 & 22.12 & 0.0053 \\
$B^{2}$ & 282.69 & 1 & 282.69 & 55.98 & 0.0007 \\
C $^{2}$ & 0.0000 & 1 & 0.0000 & 0.0000 & 1.0000 \\
Residual & 25.25 & 5 & 5.05 & & 0.1164 \\
Lack of fit & 23.25 & 3 & 7.75 & 7.75 & Not significant \\
Pure error & 2.00 & 2 & 1.0000 & & \\
Cor Total & 3558.93 & 14 & & & \\
\hline
\end{tabular}




\section{Statistics Graph (1 measurements)}

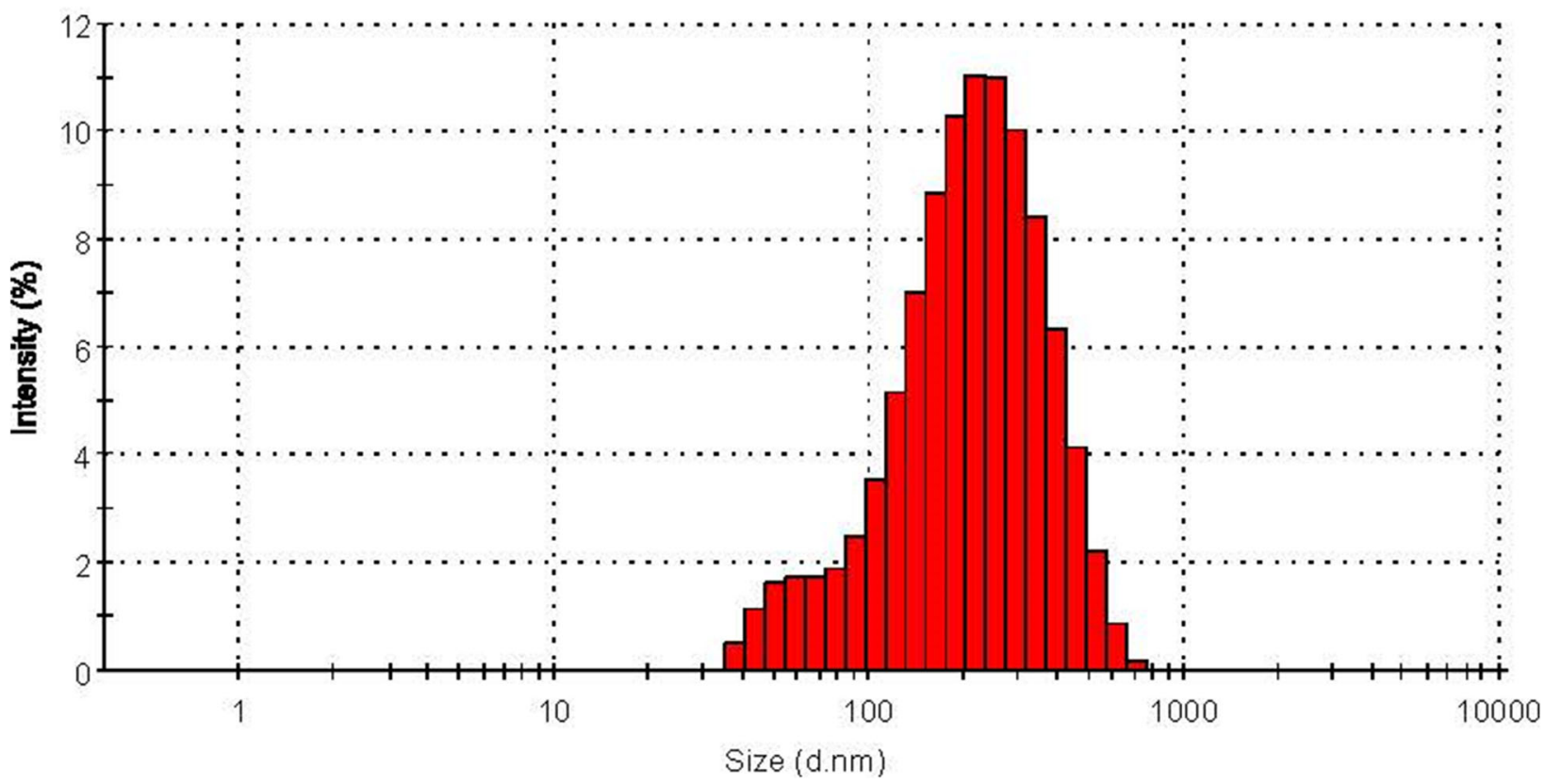

Fig. 2 Particle size of optimized formulation

\section{Entrapment efficiency}

The 1018.11 F-value obtained via design expert showed that the model was significant. In this case, the model expression $A, B, C, A B, A C, B C, B^{2}, C^{2}$ are significant, as shown in Table 5 . The lack of fit has an $F$-value of 0.00 , indicating that it is insignificant to the pure error. The predicted $R^{2}$ of 0.9988 agrees with the adjusted $R^{2}$ of 0.9989 rationally. For this model, the polynomial equation obtained is as follows:

Entrapment efficiency $=+72.33+10.50 \times \mathrm{xA}-5.00 \mathrm{xB}-$ $4.00 \times \mathrm{C}+0.5000 \times \mathrm{AB}-0.5000 \times \mathrm{AC}+0.5000 \times \mathrm{xC}$ $-0.4167 x \mathrm{~A}^{2}-0.9167 \mathrm{xB}^{2}+1.08 \times \mathrm{C}^{2}$.

According to the equation above, chitosan concentration positively affects entrapment efficiency, as shown in Fig. 6. Furthermore, the coefficients $A$ and $A B$ were positive, indicating that entrapment efficiency will increase along with an increase in the concentration of chitosan and the interaction between two polymers.

\section{Optimization and validation of the model}

The optimized nano-formulation was sensibly selected based on measures such as particle size minimization and maximum entrapment efficiency. Independent factors were derived from statistical and graphical analysis based on the desired range of response values. The Design Expert Software point prediction was used to select an optimized chitosan-CMI nanoparticle formulation based on the desirability factor's proximity to 1 [23]. The selected formulation had $0.2 \%$ chitosan concentration $(A), 0.08 \%$ CMI concentration $(B)$, and $0.1 \%$ drug concentration $(C)$. The dependent variables' predicted values were within the desired range for the above composition (average particle size $185.02 \mathrm{~mm}$, percent EE $88.30 \%$ ). The experimental batch of the checkpoint utilizing the projected independent factors was developed and characterized for the response variables. The projected results and the obtained result (average particle size $182.60 \mathrm{~mm}$, percent EE $87.45 \%)$ are in good agreement, representing the optimized nano formulation's rationality.

\section{Fourier-transform infrared (FTIR) spectroscopy}

The FTIR spectroscopic analysis was used to prove the drugdrug interaction of chitosan/CMI in the samples. IR spectra of 5-ASA (mesalamine), chitosan, CMI, and drug-decorated chitosan-CMI nanoparticles are shown in Fig. 7. As shown in the figure, mesalamine was successfully loaded onto chitosan-CMI nanoparticles. The $\mathrm{O}-\mathrm{H}$ group of stretching vibrations are responsible for mesalamine peaks such as $3408 \mathrm{~cm}^{-1}$ (because of $\mathrm{O}-\mathrm{H}$ stretching), $1741 \mathrm{~cm}^{-1}$ (because of $\mathrm{C}=\mathrm{O}$ stretching), $1618,1448 \mathrm{~cm}^{-1}$ (because of $\mathrm{C}=\mathrm{C}$ and $\mathrm{C}-\mathrm{C}$ stretching), and $1618,1448 \mathrm{~cm}^{-1}$ (due to $\mathrm{C}=\mathrm{C}$ and $\mathrm{C}-\mathrm{C}$ stretching) [24]. The extended vibration group $\mathrm{O}-\mathrm{H}$ was responsible for the peaks of chitosan at 3500 to $3300 \mathrm{~cm}^{-1}$. The protonated amino (NH2) group's N-H bending vibration has a wide peak around $3278 \mathrm{~cm}^{-1}$ from the $\mathrm{O}-\mathrm{H}$ widening in the polysaccharide backbone of allied glucose and 


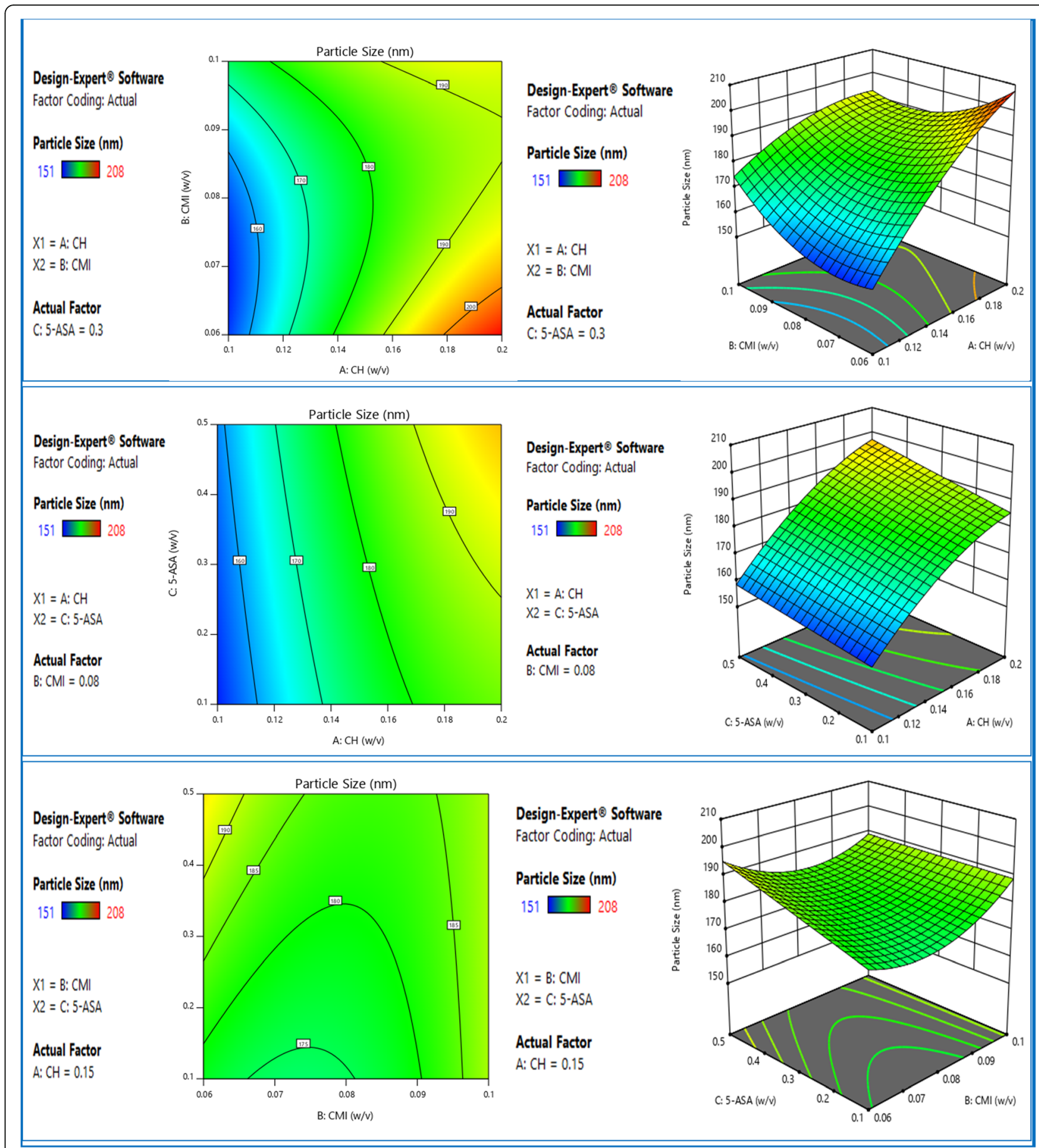

Fig. 3 2D-contour plots and 3D-response surface plots presenting the effect of independent variable on response particle size

fructose elements, and the alkyl group [25] and CMI's C-H bending vibration has a broad peak centered at $3277 \mathrm{~cm}^{-1}$ from the $\mathrm{O}-\mathrm{H}$ stretching in the polysaccharide backbone of associated glucose and fructose units. Peaks represent $\mathrm{C}-\mathrm{H}$ stretch at $2943 \mathrm{~cm}^{-1} / 2887 \mathrm{~cm}^{-1}$, and the peak at $1654 \mathrm{~cm}^{-1}$ represents the hydroxyl bending mode. Around 1037 and $932 \mathrm{~cm}^{-1}$, the bands relate to C-O-C widening, and at 1598 $\mathrm{cm}^{-1}$ and $1423 \mathrm{~cm}^{-1}$ respectively, two additional peaks are found. The carboxylate group (COO-) is responsible for the above peaks by the anti-symmetric and symmetric extending modes, which are strong evidence of the addition of inulin to carboxymethyl groups [26]. At comparable wave numbers, these peaks appeared clearly in the chitosan-CMI nanoparticle spectrum. The drug peak was not very projecting in the 
Table 4 Zeta potential and polydispersity index (PDI) of all the formulation

\begin{tabular}{lll}
\hline Formulation code & Zeta potential (mv \pm SD) & Polydispersity index \\
\hline F1 & $35.46 \pm 1.3$ & $0.232 \pm 0.02$ \\
F2 & $36.58 \pm 1.0$ & $0.248 \pm 0.04$ \\
F3 & $28.36 \pm 1.5$ & $0.355 \pm 0.08$ \\
F4 & $22.36 \pm 2.0$ & $0.355 \pm 0.10$ \\
F5 & $25.98 \pm 3.4$ & $0.245 \pm 0.12$ \\
F6 & $27.26 \pm 1.6$ & $0.242 \pm 0.05$ \\
F7 & $32.58 \pm 2.6$ & $0.238 \pm 0.03$ \\
F8 & $30.02 \pm 2.3$ & $0.242 \pm 0.14$ \\
F9 & $24.60 \pm 4.2$ & $0.247 \pm 0.12$ \\
F10 & $24.08 \pm 2.2$ & $0.350 \pm 0.06$ \\
F11 & $18.98 \pm 3.2$ & $0.262 \pm 0.02$ \\
F12 & $20.36 \pm 3.0$ & $0.242 \pm 0.07$ \\
F13 & $26.54 \pm 2.8$ & $0.238 \pm 0.15$ \\
F14 & $36.20 \pm 1.5$ & $0.251 \pm 0.13$ \\
F15 & $30.24 \pm 1.8$ & $0.255 \pm 0.05$ \\
\hline
\end{tabular}

formulation because it could be available in the polymer matrix as molecular dispersion. The FTIR findings indicate that the polymer and the drug were not chemically incompatible.

\section{Differential scanning calorimeter (DSC)}

The DSC thermogram shows an endothermic melting peak of pure mesalamine, representing its crystalline nature, at $284{ }^{\circ} \mathrm{C}$ [27]. The crystalline matter of chitosan, and the amount of water loss associated with the polymer's hydrophilic groups, have a sharp endothermic peak at $316^{\circ} \mathrm{C}$ [28]. Furthermore, the melting of different semi-crystalline isoforms causes endothermic transitions between 167 and $185^{\circ} \mathrm{C}$ in the carboxymethyl inulin sample [29], with a glass transition occurring at $167^{\circ} \mathrm{C}$ before degradation at $240^{\circ} \mathrm{C}$. The calorimetry of nanoparticles showed that the mesalamine peak shifted from 284 to $280^{\circ} \mathrm{C}$, showing a decrease in the temperature of its glass transition (Tg) (Fig. 8) [30].

\section{In vitro drug release profile of developed chitosan-CMI nanoparticles}

In vitro release finding on mesalamine-loaded nanoformulations at $\mathrm{pH} 7.4$ followed by $\mathrm{pH} 1.2$ revealed that drug release was minimal (about 5\%) in the acidic $\mathrm{pH}$, even though the drug release increased exponentially after a few hours in $7.4 \mathrm{pH}$ buffer (up to $84 \%$ ) until $24 \mathrm{~h}$, as shown in Fig. 9. Negligible drug release in the acidic media may be due to the enzyme-sensitive polymer inulin, which was adsorbed on the nanoparticle surface, and minimal hydration of the formulation. At higher $\mathrm{pH}$, chitosan present in the nanoparticle forms an interpenetrating network that, when contacted with alkaline media, swells and forms a dense, rigid network, leading to increased diffusion path length through which leaching of the drug occurs slowly, showing a sustained release pattern [31]. Figure 9 shows the comparative dissolution profile of mesalamine marketed tablet "PENTASA" $500 \mathrm{mg}$ and optimized chitosan-CMI nanoparticle.

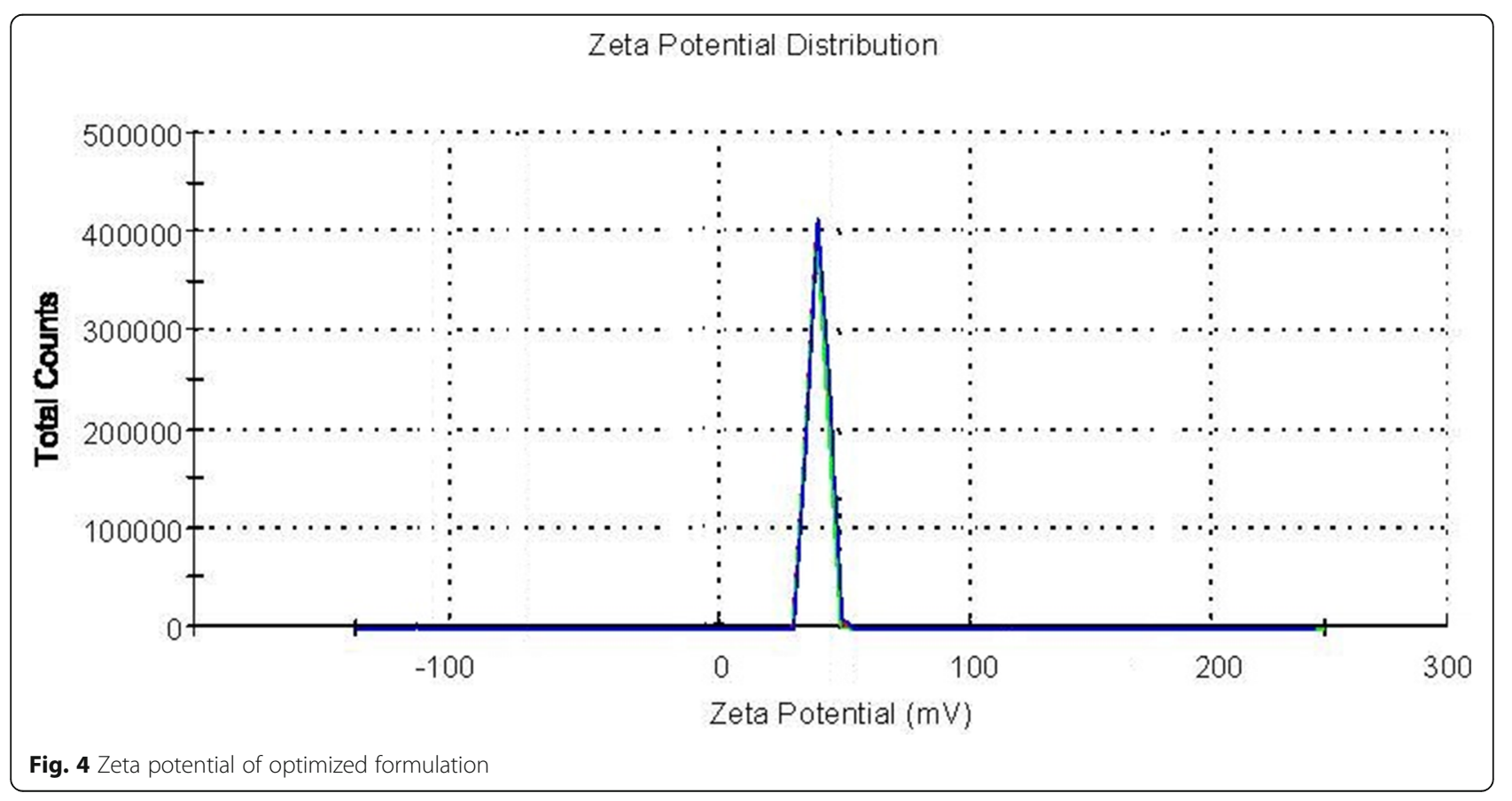




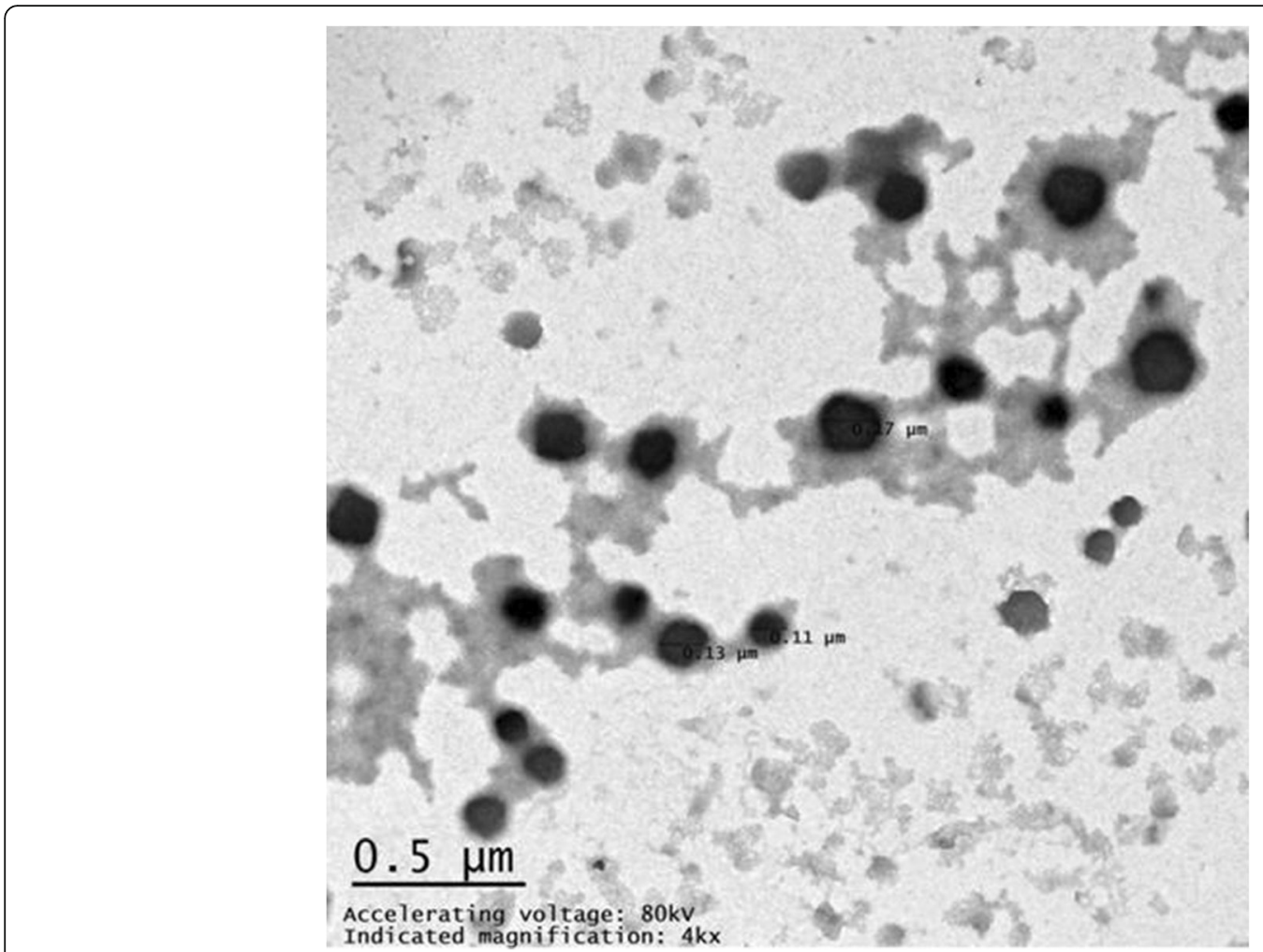

Fig. 5 TEM image of optimized formulation

The mesalamine of conventional tablet showed release with a percent cumulative drug release (\% CDR) of $57.91 \%$ in $24 \mathrm{~h}$ whereas the optimized nanoparticles exhibited \% CDR of $84.43 \%$ in $24 \mathrm{~h}$. Hence, it can be concluded that the prepared nanoparticles show better-sustained release behavior compared to conventional marketed tablets.

\section{Kinetic modeling analysis}

In order to select the optimal fit model and regression coefficient, in vitro release data was incorporated into different mathematical models, including zero-order, first order, Higuchi Matrix, Korsemeyer-Peppas and Hixson Crowell. Table 6 displays the results obtained In the case of Higuchi and Korsemeyer-Peppas, the regression

Table 5 Statistical analysis result of entrapment efficiency

\begin{tabular}{llllll}
\hline Source & Sum of squares & df & Mean square & $\boldsymbol{F}$-value & $\boldsymbol{p}$-value \\
\hline Model & 1221.73 & 9 & 135.75 & 1018.11 & $<0.0001$ \\
A-CH & 882.00 & 1 & 882.00 & 6615.00 & $<0.0001$ \\
B-CMI & 200.00 & 1 & 200.00 & 1500.00 & $<0.0001$ \\
C-5-ASA & 128.00 & 1 & 128.00 & 960.00 & $<0.0001$ \\
AB & 1.0000 & 1 & 1.0000 & 7.50 & 0.0409 \\
AC & 1.0000 & 1 & 1.0000 & 7.50 & 0.0409 \\
BC & 1.0000 & 1 & 1.0000 & 7.50 & 0.0409 \\
$A^{2}$ & 0.6410 & 1 & 0.6410 & 4.81 & 0.0798 \\
$B^{2}$ & 3.10 & 1 & 3.10 & 23.27 & 0.0048 \\
$C^{2}$ & 4.33 & 1 & 4.33 & 32.50 & 0.0023 \\
Residual & 0.6667 & 5 & 0.1333 & & 1.0000 \\
Lack of fit & 0.0000 & 3 & 0.0000 & 0.0000 & Not significant \\
Pure error & 0.6667 & 2 & 0.3333 & & \\
Cor Total & 1222.40 & 14 & & & \\
\hline
\end{tabular}




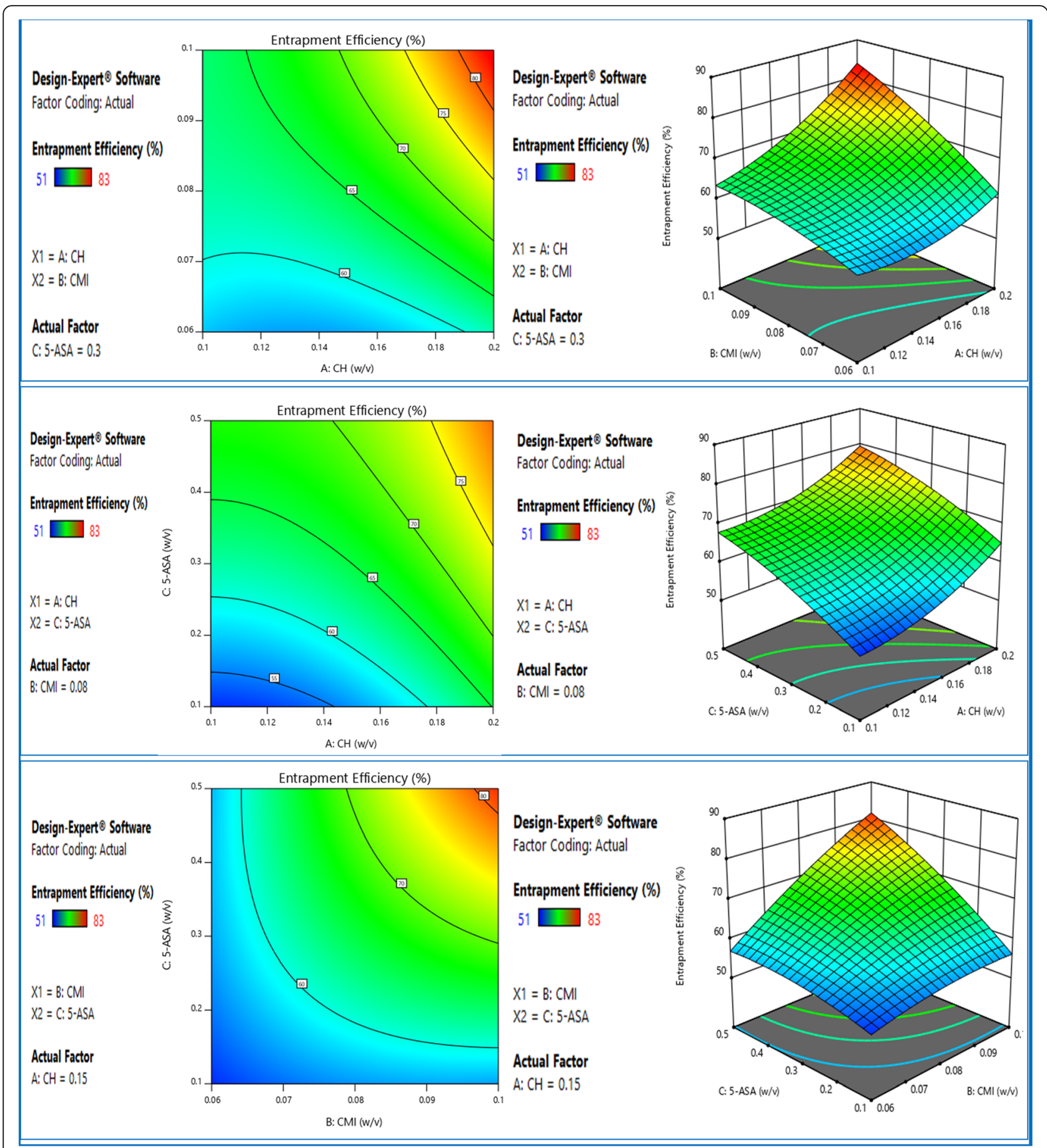

Fig. 6 2D-contour plots and 3D-response surface plots presenting the effect of independent variable on entrapment efficiency

coefficient $\left(R^{2}\right)$ finding of 0.9426 of the kinetic model for prepared formulation referred to above show values close to one, the additional $R^{2}$ values of 0.9784 are very close to one, and the ploy reveals linearity. This result supports the formulation's sustained release behavior. The $n$ value in the optimized formulation is 0.6911 . As a result, it is concluded that the optimized formulation used a non-Fickian release pattern, in which release was controlled by means of diffusion and swelling, as described by the Higuchi and Korsemeyer-Peppas models.

\section{Ex vivo mucoadhesion}

The results obtained from the ex vivo mucoadhesive study are presented in Fig. 10. The result showed that 


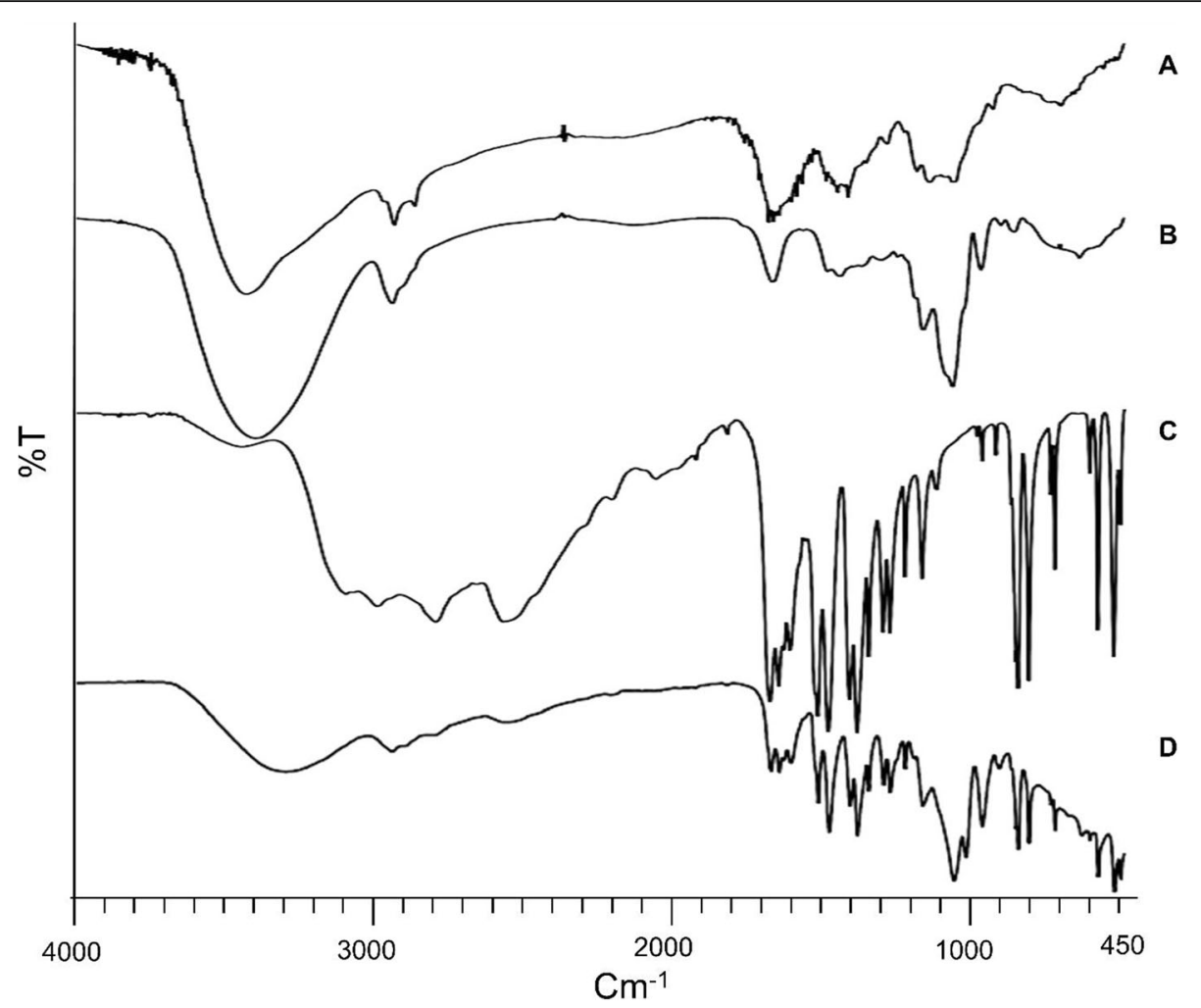

Fig. 7 FTIR spectra of (A) chitosan, (B) CMI, (C) mesalamine, and (D) chitosan-CMI nanoparticle

the mucoadhesion of chitosan-CMI nanoparticle with colonic mucosa is due to the ionic association among the positively charged chitosan and negatively charged sialic acid of mucin facilitates the attraction of nanoformulation with the mucus layer of colonic epithelium, resulting in mucoadhesion. The interaction between the chitosan-CMI nanoparticles and the colonic mucosa was confirmed using fluorescence microscopy, elucidating nanoparticle binding sites all over the body.

\section{Discussion}

Entrapment efficiency and particle size are critical considerations for developing effective therapeutic drug delivery systems to ensure that the desired amount of drugs is delivered to the target site for the optimum therapeutic response. These attributes can be managed during the initial phase of development by analyzing some formulation and process parameters [32, 33]. BBD has been used in this work to optimize the nanoparticles developed with Chitosan-CMI. The results of the BBD were analyzed, and the utility of this statistical design resulted in a significant amount of data being provided to optimize the formulation. All responses were adjusted to fit the quadratic model, and ANOVA was used to check for model compatibility, lack of fit, and regression coefficients $\left(R^{2}\right)$. Each response should be connected to the others to optimize responses, and a most supportive zone should be required for each response to eliminate bias. Many types of literature have supported the desirability function to optimize multiple responses [34, 35].

The FTIR study revealed that mesalamine was successfully loaded onto chitosan-CMI nanoparticles. All prominent drug peaks were detected in the optimized formulation, indicating that the drug and polymers used in the formulation have no chemical interaction. The endothermic melting peak of pure mesalamine, which represents its crystalline nature, is $284^{\circ} \mathrm{C}$ [36]. DSC study also confirms that there was no drug-polymers interaction among the formulation. The thermal peak of the optimized formulation illustrated a wide melting peak $\left(284-292^{\circ} \mathrm{C}\right)$. This indicates that mesalamine was converted to an amorphous form. The rationale of the observation may be the entrapment of the drug in the polymer matrix [37].

As the polymer concentration increases, the organic phase's viscosity rises, slowing the rate of diffusion towards the aqueous phase. Diffusion is slowed, which encourages the formation of large nanodroplets at the interface [38]. The mean diameter of nanoparticles against polymer concentration was close to linearity with $R^{2}=0.9929$. These findings support previous research [39], which found that the higher the polymer concentration in the sample, the higher the frequency of collision, the higher the 

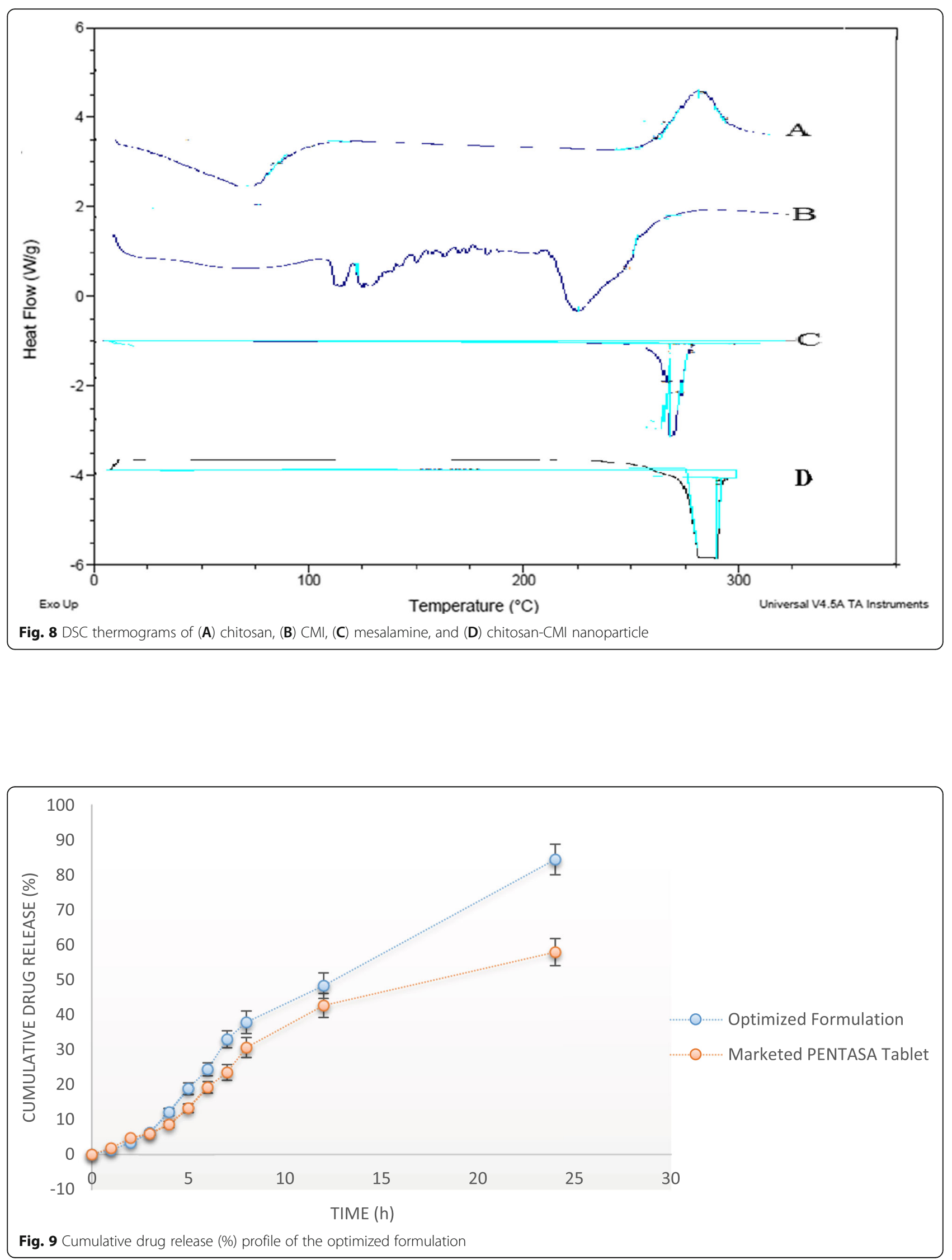
Table 6 Model fitting results of optimized chitosan-CMI nanoparticle

\begin{tabular}{llllll}
\hline $\begin{array}{l}\text { Optimized } \\
\text { formulation } \\
\text { code }\end{array}$ & \multicolumn{2}{l}{ Regression coefficient $\boldsymbol{R}^{2}$} & $\begin{array}{l}\text { Release } \\
\text { exponent } \\
(\boldsymbol{n})\end{array}$ \\
\cline { 2 - 6 } & Zero order & First order & Higuchi & Peppas \\
\hline OF1 & 0.9845 & 0.8935 & 0.9426 & 0.9784 & 0.6911 \\
\hline
\end{tabular}

concentration of semi-formed particles, and the larger the overall size of the nanoparticles. The morphological study performed with the help of TEM showed that the particles were nearly spheroidal in shape and were all approximately the same in size. The zeta potential of nanoparticles is affected by the polyelectrolyte ratio, with a decrease in chitosan viscosity causing structural instability and thus lowering the zeta potential [40]. A positive charge value was found in all nanoparticles, also with a maximum charge value of 36.46 , and a PDI of 0.248 indicates good dispersion stability and homogeneity [22]. This positive surface charge causes epithelial mucin interaction, which has mucoadhesion characteristics [41]. By increasing the concentration of chitosan at the lower CMI level, the entrapment efficiency was improved. Similarly, the efficiency of entrapment improves with increasing CMI, but not in a linear fashion. These findings suggest that increasing chitosan concentration leads to increased encapsulation efficiency. This has confirmed the findings of previous studies, which have shown that chitosan has a rapid gel-forming capability and a high viscosity resulting in a more robust hydrogel matrix and optimal entrapment $[42,43]$.

The result of cumulative drug release for optimized chitosan-CMI nanoparticles over $24 \mathrm{~h}$ in simulated gastric fluid (SGF) and in SCF showed that the formulation released less than $5 \%$ of the drug after $2 \mathrm{~h}$ in an acidic environment; it is recommended that the formulation's drug release be minimized in the upper gastro-intestinal region, which was achieved in the developed chitosanCMI formulation. The drug release profile was gradually increased after the acidic condition and was found to be $60.32 \pm 1.74$ at the end of $12 \mathrm{~h}$. The next aliquote was taken after $24 \mathrm{~h}$, and the release of the drug was reported to be $82.87 \pm 1.80$. Since chitosan and CMI have different pKa values, they respond differently in the context of protonation/deprotonation as a $\mathrm{pH}$ function. Both the $\mathrm{NH}_{2}{ }^{+}$group of chitosan and $\mathrm{COO}^{-}$groups of CMI are protonated under acidic conditions ( $\mathrm{pH}$ 1.2). The ionization of CMI carboxylic groups is partial and prevails, which helps to protect the nanoparticles through the coulombic repulsion of carboxylic groups. Inulin is a natural polysaccharide hydrolyzed by inulinase produced by the microbiome in the colon but not by GI digestive enzymes [44]. However, mild swelling and minimal mesalamine leaching were noted at $1.2 \mathrm{pH}$. At the colonic region $(7.4 \mathrm{pH})$, CMI carboxylic groups get deprotonated, resulting in electrostatic repulsion between ionized groups, giving rise to chain repulsion, and chitosan in the nanoparticle get swell and form a dense, rigid network leading to slow leaching of the drug showing sustained release pattern [31]. The release kinetics applied on different kinetic models revealed a non-Fickian release mechanism, i.e., the release was regulated by diffusion-controlled and swelling-controlled mechanisms. The developed chitosan-CMI nanoparticle showed good mucoadhesion with colonic mucosa; this may be due to ionic interaction among the positively charged chitosan and negatively charged sialic acid of mucin [45].

\section{Conclusion}

This work has successfully synthesized and characterized a novel, and effective drug nanocarrier focused on ionotropic gelation of the biocompatible, biodegradable, nontoxic biopolymers such as chitosan and CMI. The effect of three independent variables on particle size and percent entrapment efficiency was studied and optimized by BBD. The nanoparticle formulation method did not involve any external cross-linking or stabilizing agents; efficient derivatizations and easy and accurate purification

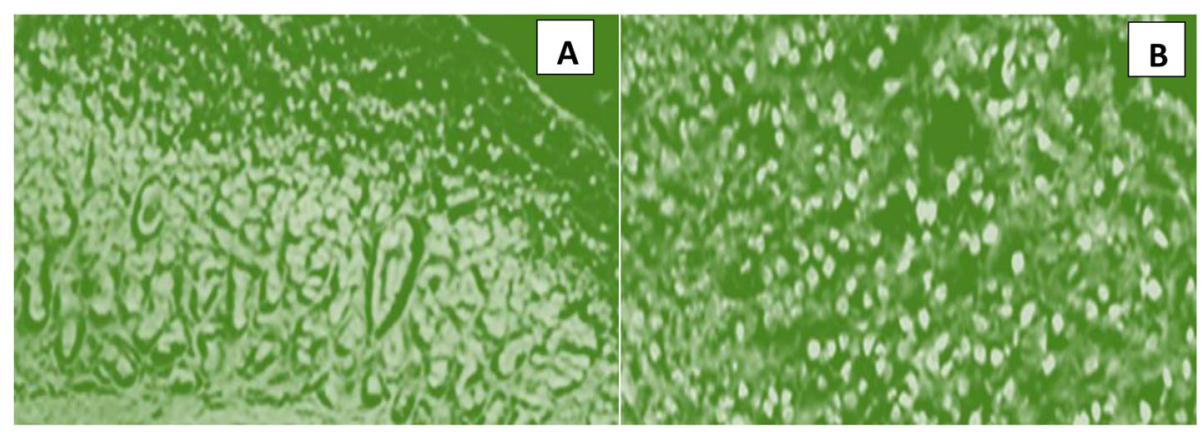

Fig. 10 A Fluorescent image of colonic mucosa without formulation. B Fluorescent image of colonic mucosa showing mucoadhesion of optimized Nanoparticulate formulation 
of the finished product allow this nanocarrier to be very appealing for colonic disorders. The resultant nanoparticles were uniform in diameter in the range of 151-208 $\mathrm{nm}$ and resistant to hydrolytic degradation. The mesalamine adducts release profile has also been investigated, with less than $5 \%$ release in the gastric environment ( $\mathrm{pH} 1.2)$. The vast majority of the medication was released in the colonic region ( $\mathrm{pH} 7.4)$, which is a highly desirable condition for effective colonic disorder management. The findings suggest that BBD can be a valuable tool for optimizing nano-formulations and that the chitosan-CMI nanoparticles can be a potential carrier for delivering mesalamine to their target sites. As a result, the proposed nano-pharmaceutical has a high chance of being used in a "bench-to-bed" study. Such research may lead to a "side effect-free" drug delivery system for the treatment of inflammatory bowel disease.

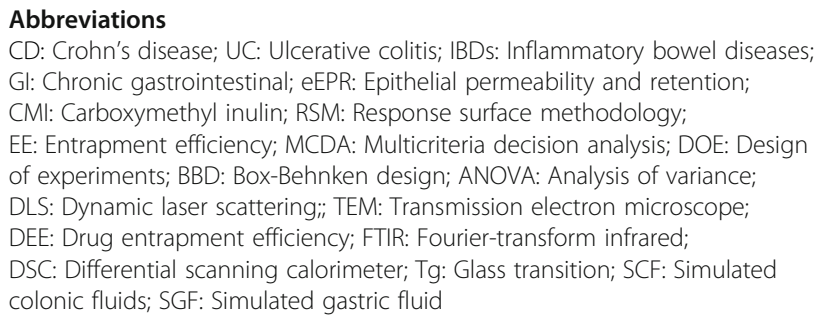

\section{Acknowledgements}

The authors express special thanks to Suman Jain (Director, School of studies in pharmaceutical sciences, Jiwaji University, Gwalior) and Mukul Tailang for his kind support (Department of Pharmaceutics, School of studies in pharmaceutical sciences, Jiwaji University, Gwalior).

\section{Authors' contributions}

"WA" designed and optimizes the study and developed the methodology. WA performed the experiments and collection and interpretation of data. WA wrote the manuscript. "NG" contributed to manuscript revision and provided supervision. WA and NG read and approved the final manuscript.

\section{Funding}

The University Grant Commission (UGC) is a funding agency which comes under the HRD ministry, Government of India. The main role of UGC in this research work was their financial support under the scheme of "Maulana Azad National Fellowship" (MANF) (201718-MANF-2017-18-MAD-85337).

\section{Availability of data and materials}

The datasets of research were collected from experiments and analysis of variables during current study. These datasets are available from the corresponding author on reasonable request.

\section{Declarations}

Ethics approval and consent to participate Not applicable.

\section{Consent for publication}

Not applicable.

\section{Competing interests}

The authors declare that they have no competing interests.
Received: 19 March 2021 Accepted: 5 July 2021

Published online: 21 July 2021

\section{References}

1. Ordás I, Eckmann L, Talamini M, Baumgart DC, Sandborn WJ (2012) Ulcerative colitis. Lancet 380(9853):1606-1619. https://doi.org/10.1016/S014 0-6736(12)60150-0

2. Baumgart DC, Sandborn WJ (2007) Inflammatory bowel disease: clinical aspects and established and evolving therapies. Lancet 369(9573):16411657. https://doi.org/10.1016/50140-6736(07)60751-X

3. Mladenovska K, Cruaud O, Richomme P, Belamie E, Raicki RS, VenierJulienne MC, Popovski E, Benoit JP, Goracinova K (2007) 5-ASA loaded chitosan-Ca-alginate microparticles: preparation and physicochemical characterization. Int J Pharm 345(1-2):59-69. https://doi.org/10.1016/j.jpha rm.2007.05.059

4. Viscido A, Capannolo A, Latella G (2014) Nanotechnology in the treatment of inflammatory bowel diseases. J Crohns Colitis 8(9):903-918. https://doi. org/10.1016/j.crohns.2014.02.024

5. Giron F, Pastó A, Tasciotti E, Abraham BP (2019) Nanotechnology in the treatment of inflammatory bowel disease. Inflamm Bowel Dis 25(12):18711880. https://doi.org/10.1093/ibd/izz205

6. Janes KA, Fresneau MP, Marazuela A, Fabra A, Alonso MJ (2001) Chitosan nanoparticles as delivery systems for doxorubicin. J Control Release 73(2-3): 255-267. https://doi.org/10.1016/S0168-3659(01)00294-2

7. Li Y, Maciel D, Rodrigues J, Shi X, Tomas H Biodegradable polymer nanogels for drug/nucleic acid delivery. Chem Rev 115(16):8564-8608

8. Collnot EM, Ali H, Lehr CM (2012) Nano-and microparticulate drug carriers for targeting of the inflamed intestinal mucosa. J Control Release 161(2): $235-246$

9. Lamprecht A, Yamamoto H, Takeuchi H, Kawashima Y (2005) Nanoparticles enhance therapeutic efficiency by selectively increased local drug dose in experimental colitis in rats. J Pharmacol Exp Ther 315(1):196-202. https://doi. org/10.1124/jpet.105.088146

10. Beloqui A, Coco R, Alhouayek M, Solinís MÁ, Rodríguez-Gascón A, Muccioli GG, Préat V (2013) Budesonide-loaded nanostructured lipid carriers reduce inflammation in murine DSS-induced colitis. Int J Pharm 454(2):775-783. https://doi.org/10.1016/j.jipharm.2013.05.017

11. Rodrigues S, Dionísio M, López CR, Grenha A (2012) Biocompatibility of chitosan carriers with application in drug delivery. J Funct Biomater 3(3): 615-641. https://doi.org/10.3390/jfb3030615

12. Santiago-Rodríguez L, Lafontaine MM, Castro C, Méndez-Vega J, LatorreEsteves M, Juan EJ, Mora E, Torres-Lugo M, Rinaldi C (2013) Synthesis, stability, cellular uptake, and blood circulation time of carboxymethyl-inulin coated magnetic nanoparticles. J Mater Chem B1 (22):2807-2817

13. Chuah LH, Billa N, Roberts CJ, Burley JC, Manickam S (2013) Curcumincontaining chitosan nanoparticles as a potential mucoadhesive delivery system to the colon. Pharm Dev Technol 18(3):591-599. https://doi.org/1 0.3109/10837450.2011.640688

14. Modaresi SMS, Ejtemaei Mehr S, Faramarzi MA, Esmaeilzadeh Gharehdaghi E, Azarnia M, Modarressi MH, Baharifar H, Vaez SJ, Amani A (2014) Preparation and characterization of self-assembled chitosan nanoparticles for the sustained delivery of streptokinase: an in vivo study. Pharm Dev Technol 19(5):593-597. https://doi.org/10.3109/10837450.2013.813542

15. Box GE, Behnken DW (1960) Some new three level designs for the study of quantitative variables. Technometrics 2(4):455-475. https://doi.org/10.1080/ 00401706.1960 .10489912

16. Ramírez-Bergeron DL, Runge A, Adelman DM, Gohil M, Simon MC (2006) HIF-dependent hematopoietic factors regulate the development of the embryonic vasculature. Dev Cell 11(1):81-92. https://doi.org/10.1016/j. devcel.2006.04.018

17. Yin Y, Chen D, Qiao M, Wei X, Hu H (2007) Lectin-conjugated PLGA nanoparticles loaded with thymopentin: ex vivo bioadhesion and in vivo biodistribution. J Control Release 123(1):27-38. https://doi.org/10.1016/j. jconrel.2007.06.024

18. Niizawa I, Espinaco BY, Zorrilla SE, Sihufe GA (2019) Natural astaxanthin encapsulation: use of response surface methodology for the design of alginate beads. Int J Biol Macromol 121:601-608. https://doi.org/10.1016/j. ijbiomac.2018.10.044

19. Akram W, Garud N (2020) Optimization of inulin production process parameters using response surface methodology. Fut J Pharmaceut Sci 6(1): $1-9$ 
20. Gan Q, Wang T, Cochrane C, McCarron P (2005) Modulation of surface charge, particle size and morphological properties of chitosan-TPP nanoparticles intended for gene delivery. Colloids Surfurface, B 44(2-3):6573. https://doi.org/10.1016/j.colsurfb.2005.06.001

21. Zhang H, Oh M, Allen C, Kumacheva E (2004) Monodisperse Chitosan Nanoparticles for Mucosal Drug Delivery. Biomacromolecules 5(6):24612468. https://doi.org/10.1021/bm0496211

22. Cegnar M, Kos J, Kristl J (2004) Cystatin incorporated in poly (lactide-coglycolide) nanoparticles: development and fundamental studies on preservation of its activity. Eur J Pharm Sci 22(5):357-364. https://doi.org/1 0.1016/j.ejps.2004.04.003

23. Verma A, Sharma G, Jain A, Tiwari A, Saraf S, Panda PK, Katare OP, Jain SK (2019) Systematic optimization of cationic surface engineered mucoadhesive vesicles employing Design of Experiment (DoE): a preclinical investigation. Int J Biol Macromol 133:1142-1155. https://doi.org/10.1016/j. ijbiomac.2019.04.118

24. Neufeld L, Bianco-Peled H (2015) Designing a biocompatible hydrogel for the delivery of mesalamine. Int J Pharm 491(1-2):170-179. https://doi.org/1 0.1016/j.jpharm.2015.06.026

25. Oh JW, Chun SC, Chandrasekaran M (2019) Preparation and in vitro characterization of chitosan nanoparticles and their broad-spectrum antifungal action compared to antibacterial activities against phytopathogens of tomato. Agronomy 9(1):21. https://doi.org/10.3390/a gronomy9010021

26. Rahul R, Jha U, Sen G, Mishra S (2014) Carboxymethyl inulin: A novel flocculant for wastewater treatment. Int J Biol Macromol 63:1-7. https://doi. org/10.1016/j.jibiomac.2013.10.015

27. Seymour RW, Cooper SL (1973) Thermal analysis of polyurethane block polymers. Macromolecules 6(1):48-53. https://doi.org/10.1021/ma60031a008

28. Shahsavari S, Vasheghani-Farahani E, Ardjmand M, Dorkoosh F (2014) Design and Characterization of Acyclovir Loaded Nanoparticles for Controlled Delivery System. Curr Nanosci 10(4):521-531. https://doi.org/1 $0.2174 / 15734137113096660128$

29. Cooper PD, Barclay TG, Ginic-Markovic M, Petrovsky N (2013) The polysaccharide inulin is characterized by an extensive series of periodic isoforms with varying biological actions. Glycobiology 23(10):1164-1174. https://doi.org/10.1093/glycob/cwt053

30. Seifirad S, Karami H, Shahsavari S, Mirabasi F, Dorkoosh F (2016) Design and characterization of mesalamine loaded nanoparticles for controlled delivery system. Nanomed Res J 1(2):97-106

31. Sinha P, Udhumansha U, Rathnam G, Ganesh M, Jang HT (2017) Capecitabine encapsulated chitosan succinate-sodium alginate macromolecular complex beads for colon cancer targeted delivery: in vitro evaluation. Int J Biol Macromol 117:840-850

32. Tefas LR, Sylvester B, Tomuta I, Sesarman A, Licarete E, Banciu M, Porfire A (2017) Development of antiproliferative long-circulating liposomes coencapsulating doxorubicin and curcumin, through the use of a quality-bydesign approach. Drug Design Dev Ther 11:1605-1621. https://doi.org/1 0.2147/DDDT.S129008

33. Akram W, Joshi R, Garud N (2019) Inulin: a promising carrier for controlled and targeted drug delivery system. J Drug Delivery Therapeut 9(1-s):437441

34. Marasini N, Yan YD, Poudel BK, Choi HG, Yong CS, Kim JO (2012) Development and optimization of self-nanoemulsifying drug delivery system with enhanced bioavailability by Box-Behnken design and desirability function. J Pharm Sci 101(12):4584-4596. https://doi.org/10.1002/ jps.23333

35. Ferreira T, Oliveira J, Caiola N, De Sostoa A, Casals F, Cortes R, Economou A, Zogaris S, Garcia-Jalon D, Ilhéu M, Martinez-Capel F (2007) Ecological traits of fish assemblages from Mediterranean Europe and their responses to human disturbance. Fish Manag Ecol 14(6):473-481. https://doi.org/10.1111/ j.1365-2400.2007.00584.x

36. Seymour RW, Estes GM, Cooper SL (1970) Infrared studies of segmented polyurethan elastomers. I Hydrogen bonding. Macromolecules 3(5):579-583

37. Yadav SK, Khan G, Bansal M, Vardhan H, Mishra B (2017) Screening of ionically crosslinked chitosan-tripolyphosphate microspheres using PlackettBurman factorial design for the treatment of intrapocket infections. Drug Dev Ind Pharm 43(11):1801-1816. https://doi.org/10.1080/03639045.2017.134 9782

38. Sharma PR, Varma AJ (2014) Thermal stability of cellulose and their nanoparticles: Effect of incremental increases in carboxyl and aldehyde groups. Carbohydr Polym 114:339-343. https://doi.org/10.1016/j.carbpol.2 014.08.032

39. Al-Kassas RS, Al-Gohary OM, Al-Faadhel MM (2007) Controlling of systemic absorption of gliclazide through incorporation into alginate beads. Int J Pharm 341(1-2):230-237. https://doi.org/10.1016/j.ijpharm.2007.03.047

40. Rampino A, Borgogna M, Blasi P, Bellich B, Cesàro A (2013) Chitosan nanoparticles: preparation, size evolution and stability. Int J Pharm 455(1-2): 219-228. https://doi.org/10.1016/j.jpharm.2013.07.034

41. Hong SC, Yoo SY, Kim H, Lee J (2017) Chitosan-based multifunctional platforms for local delivery of therapeutics. Marine Drugs 15(3):60. https:// doi.org/10.3390/md15030060

42. Elmizadeh $H$, Khanmohammadi M, Ghasemi K, Hassanzadeh G, Nassiri-Asl M, Garmarudi AB (2013) Preparation and optimization of chitosan nanoparticles and magnetic chitosan nanoparticles as delivery systems using BoxBehnken statistical design. J Pharm Biomed Anal 80:141-146. https://doi. org/10.1016/j.jpba.2013.02.038

43. Joshi R, Garud N (2021) Development, optimization and characterization of flurbiprofen matrix transdermal drug delivery system using Box-Behnken statistical design. Fut J Pharmaceut Sci 7(1):1-8

44. Wan X, Guo H, Liang Y, Zhou C, Liu Z, Li K, Niu F, Zhai X, Wang L (2020) The physiological functions and pharmaceutical applications of inulin: A review. Carbohydr Polym 116589

45. Alkhader E, Billa N, Roberts CJ (2017) Mucoadhesive chitosan-pectinate nanoparticles for the delivery of curcumin to the colon. AAPS PharmSciTech 18(4):1009-1018. https://doi.org/10.1208/s12249-016-0623-y

\section{Publisher's Note}

Springer Nature remains neutral with regard to jurisdictional claims in published maps and institutional affiliations.

\section{Submit your manuscript to a SpringerOpen ${ }^{\circ}$ journal and benefit from:}

- Convenient online submission

- Rigorous peer review

- Open access: articles freely available online

High visibility within the field

- Retaining the copyright to your article

Submit your next manuscript at $\boldsymbol{\nabla}$ springeropen.com 